\title{
Lysulin: Natural nutritional support to bring better health to people with diabetes and prediabetes
}

\author{
John F. Burd, PhD \\ Lysulin, Inc., 3225A Del Mar Heights Road, Suite 398, San Diego, CA 92130 \\ Corresponding Author: John F. Burd, Lysulin, Inc. 3225A Del Mar Heights Road, Suite 398, \\ San Diego, CA 92130
}

Submission Date: March $2^{\text {nd }}, 2019$. Acceptance Date: June $28^{\text {th }}, 2019$. Publication Date: June $30^{\text {th }}, 2019$.

Citation: Burd J.F. Lysulin: Natural Nutritional Support to Bring Better Health to People with Diabetes and Prediabetes. Bioactive Compounds in Health and Disease 2019; 2(6):149-154.

https://doi.org/10.31989/bchd.v2i6.600

\begin{abstract}
Obesity and type 2 diabetes, both worldwide epidemics, are drastic problems that can be ameliorated dramatically through the combination of lifestyle changes (especially diet and exercise) the treatment of drugs and nutritional supplements [1]. Lysulin is a patent-pending nutritional supplement that contains lysine, zinc and vitamin C [2]. Through double-blind placebocontrolled studies, it has been shown to help people with prediabetes [3] and type 2 diabetes [4] in promoting better glycemic control and lowering their HbAlc.

Historically, supplements have had a bad reputation due to aggressive marketing of unsubstantiated claims. That being said, Lysulin is firmly grounded on a foundation of 25 years of research, development and clinical studies. These studies show the ingredients in Lysulin can lower blood glucose and glycated proteins - the cause of diabetes complications. In addition, double-blind placebo-controlled studies found that Lysulin improves glycemic control in people with diabetes and may also slow or even halt the progression from prediabetes to type 2 diabetes $[3,4]$.

Glucose is essential in providing the human body an energy source. Glucose relies on the hormone, insulin, to enter our cells to provide the energy we need for everyday living. However, while everyone needs a certain amount of glucose for daily energy production, excessive glucose is dangerously toxic to the body (primarily as a result of protein glycation). In addition, fructose (from High Fructose Corn Syrup) does not utilize insulin to enter the liver or our cells, and thus enters them easily and immediately turns into fat [5]. Poor diets, along with increased consumption of High Fructose Corn Syrup (HFCS), have lead to the high rates of obesity and diabetes that we see today. The pandemic of type 2 diabetes, which is expected to affect at least 250 million people worldwide by 2020 and 642 million by 2040 [6], is the result of diets high in carbohydrates, HFCS and sugar, along with the lack of commensurate exercise. This lifestyle leads to insulin resistance, caused by the glycation of insulin and insulin receptors [7], which results in high concentrations of glucose in the bloodstream.
\end{abstract}


The therapy for type 2 diabetes begins with attempts to control glucose through diet and exercise. If this fails, oral drugs are prescribed. If these fail to show improvements in blood glucose levels, insulin injections are given. Over 30\% of people with type 2 diabetes are using insulin injections to control their blood glucose levels [6]. There is vast literature documenting the role of nutraceuticals and functional foods in managing the alterations in metabolism and improving the health of people with chronic disease [8,9]. To make up for the fact that important nutrients are lost during the processing of food, adding nutritional supplements to our daily routine, in addition to consuming a low-carbohydrate diet, can have profound benefits. A well-balanced diet, along with exercise and the right nutritional supplements, can provide the foundation for a lifetime of well-being and good health.

Keywords: Diabetes, Nutritional Support, Lysulin, Lysine, Zinc, Vitamin V, HbA1c

\section{INTRODUCTION}

\section{Protein Glycation}

Glucose, because it is a reactive chemical, can be toxic in high amounts. It is an aldehyde that reacts with the amino-groups found on the amino acids of all proteins. When glucose reacts with these amino-groups, it forms a fructosamine bond, and the protein is said to be "glycated". Glycated haemoglobin, or $\mathrm{HbA1c}$, is commonly used as a diagnostic test for diabetes. Increased levels of $\mathrm{HbA1c}$ are associated with a higher risk of developing diabetes-related issues. The glycated proteins can progress through a series of reactions to become Advanced Glycation End products or AGEs (10). The chemistry of AGE formation has been well documented (11). AGEs are believed to be responsible for many, and perhaps all, of the disease complications associated with diabetes. These include retinopathy, nephropathy and neuropathy (which leads to blindness, kidney failure, organ degradation, and amputations.) (11). The glycation of insulin and the insulin receptors on our cells leads to insulin resistance and, in turn, insulin depletion [7]. Figure 1 illustrates this process of glycation.

Figure 1. Glycation of Insulin and Insulin Receptors. Top panel - normal situation. Bottom panelinsulin resistance
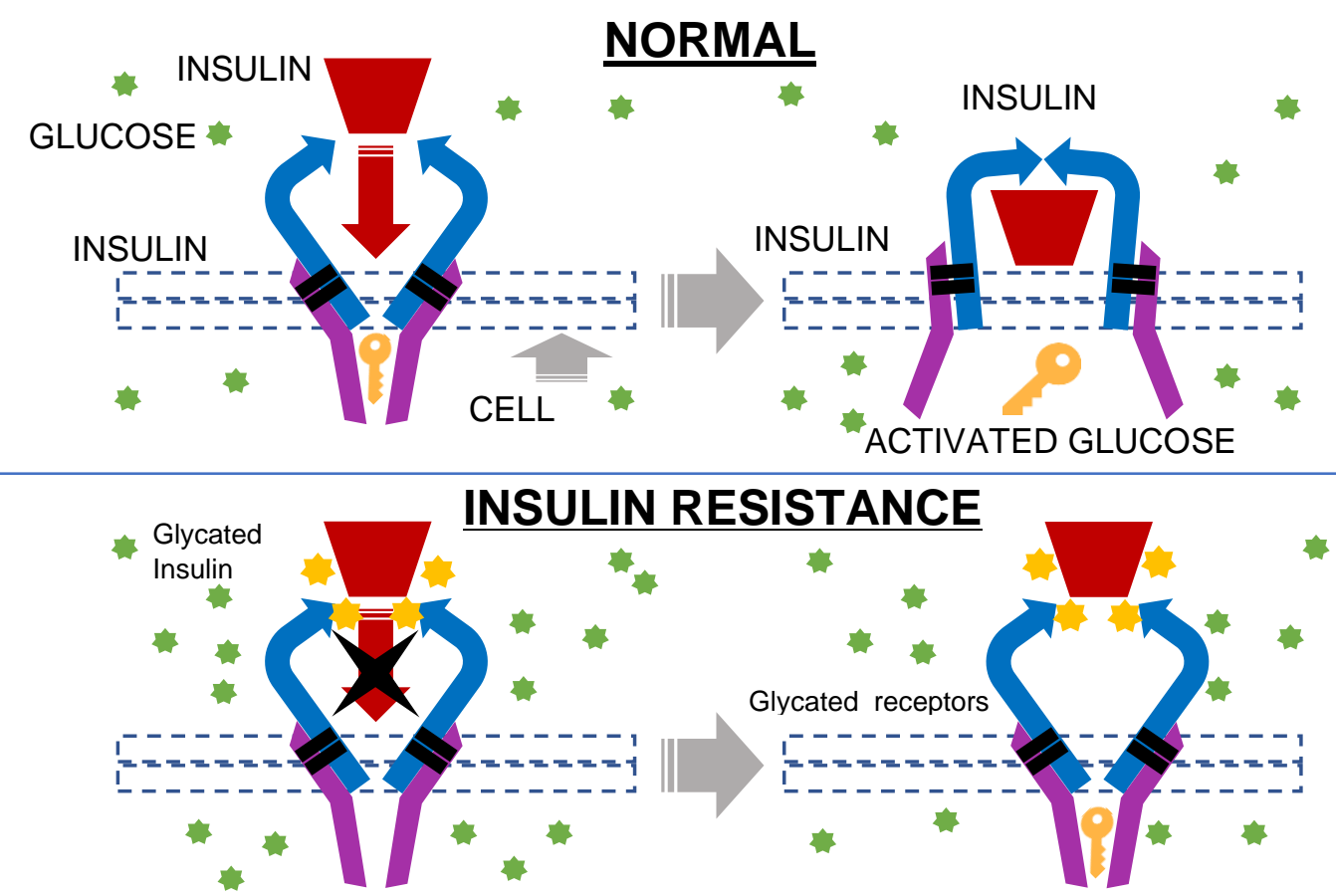

GLUCOSE KEY NOT ACTIVATED 
This logically leads to the observation that if protein glycation could be slowed or halted, the complications of diabetes could also be reduced or stopped altogether. Lysine, one of the major components of Lysulin, has been shown in diabetic animal modles to halt the production of AGEs $[12,13]$. As a result, Lysulin is considered a possible remedy that could slow/halt both the complications of type 2 diabetes and the progression of prediabetes to type 2 diabetes [3,12].

\section{Insulin depletion}

In normal individuals, insulin production can respond to the insulin needs for the entirety of a person's life. With chronic hyperglycemia, though, the pancreas makes additional insulin in an attempt to normalize blood glucose. When burdened with insulin resistance, the pancreas is called upon constantly to make more and more insulin in a heroic attempt to lower blood glucose. Persistently high blood glucose levels (i.e. glucose toxicity) eventually exhaust the ability of the pancreas to make additional insulin. When the pancreas can no longer make insulin or adequate amounts of it, a person must take insulin shots to make up for the shortfall. This calls into question the use of sulfonylurea drugs, which force the pancreas to secrete more insulin despite the fact that the patient's cells cannot properly recognize the insulin due to insulin resistance.

\section{The all-natural solution to the problem of glucose toxicity}

The issue of glucose toxicity should be a primary concern in looking at ways to prevent and treat type 2 diabetes and the complications and associated diseases that come along with it. A promising remedy to inhibiting protein glycation is the nutritional supplement with the trade name Lysulin ${ }^{\circledR}$. Lysulin contains lysine, zinc and vitamin C [2]. Earlier publications have provided detailed information on why these compounds are helpful in glucose control and diabetes management [2]. Lysulin has been shown to halt AGE production in diabetic rat studies [12]. In addition, recent double-blind placebo-controlled studies have shown that Lysulin can lower HbA1c in as little as two weeks $[14,15]$. Lysulin contains a safe, but relatively large amount of the amino acid lysine, so it blocks protein glycation as illustrated in Figure 2.

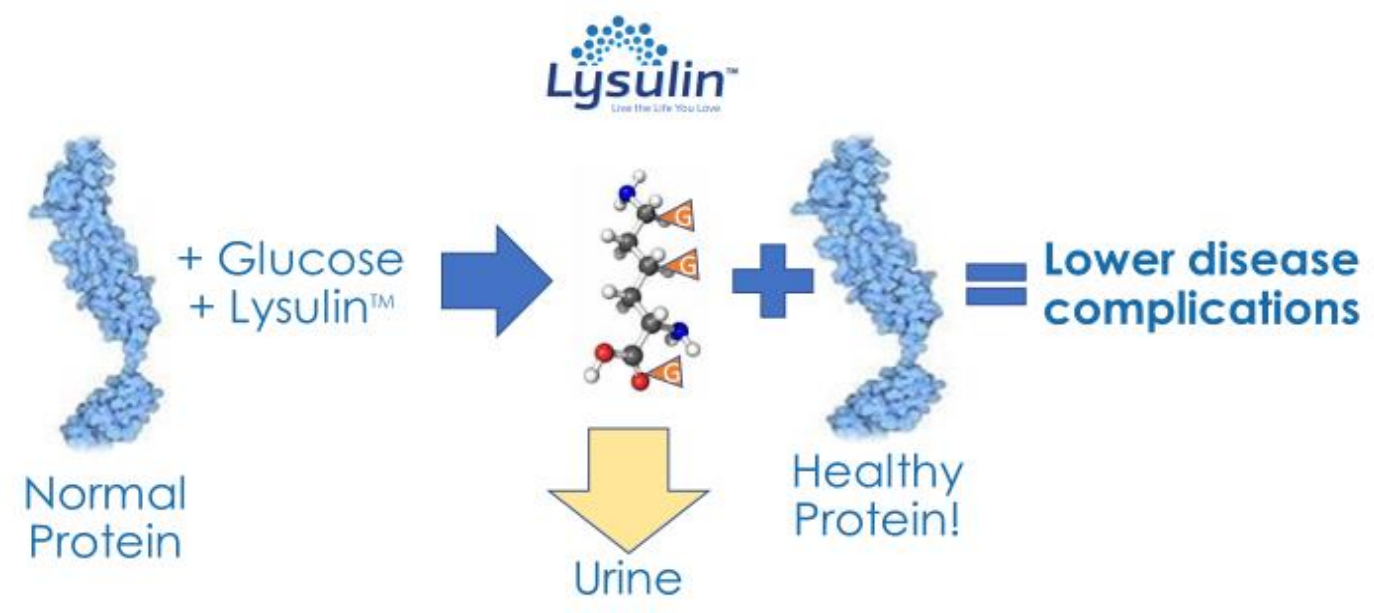

Figure 2. Mechanism for Inhibition of Protein Glycation by Lysulin. 
The lysine in Lysulin reacts with glucose and thus prevents the glycation of proteins. The glycated lysine is then safely excreted in the urine. Lysulin also contains zinc and vitamin C, both of which have been shown to lower blood glucose and prevent the progression of prediabetes to Type 2 diabetes. They have also been shown to improve insulin resistance and the general lipid profile $[16,17]$. The combination of zinc and vitamin $C$ in addition to lysine helps contribute to the lowering of $\mathrm{HbA1c}$ for patients with prediabetes [3] and type 2 diabetes [4].

\section{HbAlc Testing in the Pharmacy or at Home}

An important blood test for measuring the effectiveness of diabetes management is the Hemoglobin A1c test (also called HbA1c, A1c and glycated hemoglobin) [18]. This test is firmly established as the gold standard for examining diabetes progression. In addition, glucose selftesting can be done with test strips or several new glucose-monitoring products that are being released [19]. While the HbA1c test is normally conducted in a clinical laboratory, it can now be done in pharmacies or even at home [5]. The testing has traditionally been ordered only annually or semi-annually, but recent studies have shown that HbA1c test results can change in less than one month after making adjustments in diabetes management [20]. This rapid change can lead to a more timely therapy modification, leading to better health.

\section{CONCLUSION}

In recent years, the epidemics of obesity and type 2 diabetes have developed largely in part due to poor diets and nutritional deficiencies. A surplus of glucose in our bloodstream, stemming from chronic and excessive carbohydrate/HFCS consumption, is proving to be highly toxic to millions of people. This has led to excessive protein glycation, AGE production, insulin resistance, insulin depletion and potentially-fatal disease complications. An all-natural nutritional supplement, Lysulin, now offers a unique, effective, and affordable way to help combat glucose toxicity and the diabetes pandemic. In addition, modern $\mathrm{HbA1c}$ testing can now be done in a pharmacy or at home to help diabetics better manager their disease in a more timely fashion.

Abbreviations: Hemoglobin A1c, HbA1c, A1c and glycated hemoglobin; High Fructose Corn Syrup, HFCS; Advanced Glycation End products, AGEs, AGE.

Competing interests: John Burd, PhD is the CEO of Lysulin, Inc. and has no other conflicts of interest in regards to this manuscript.

Authors contributions: John Burd, PhD, performed the literature search, conceived of this idea, and wrote the manuscript and has the primary responsibility for the final content. Dr. Burd read, edited and approved the final manuscript.

Acknowledgements and funding: This article and the Lysulin studies were funded by Lysulin, Inc. There was no other support by any other organization or funding agency. 


\section{REFERENCES}

1. O'Rahilly, S: Science, medicine and the future. Non-insulin dependent diabetes mellitus: the gathering storm. BMJ. 1997 314: 955-959.

2. Burd, J: Lysulin ${ }^{\mathrm{TM}}$, a new supplement for Nutritional Support for People with Diabetes and Pre-diabetes (those at risk of developing diabetes). Diabetes Management; 2018: 8(2): $38-40$.

3. Ranasinghe $\mathrm{P}$, et al.: Zinc supplementation in prediabetes: A randomized double-blind placebo-controlled clinical trial. J. Diabetes 2017 10, 386-397.

4. Burd JF, Noetzel V, Gonzalez A and Melero FAA: Lysulin®: A double-blind placebo controlled pilot study of daily oral supplementation in people with type 2 diabetes. Diabetes Management (2018) 8(6), 154-162.

5. Parks E. J., Skokan, L.E. Timlin, M T, Dingfelder C.S: Dietary sugars stimulate fatty acid synthesis in adults. J. Nutr. 2008 138: 1039-1046.

6. Centers for Disease Control and Prevention (CDC), National Center for Health Statistics, Division of Health Interview Statistics, data from the National Health Interview Survey. Data analyzed by personnel in the CDC's Division of Diabetes Translation, National Center for Chronic Disease Prevention and Health Promotion.

7. Rhinesmith T, Turkete T, Root-Bernstein R: Rapid Non-Enzymatic Glycation of the Insulin Receptor under Hyperglycemic Conditions Inhibits Insulin Binding In Vitro: Implications for Insulin Resistance. Int J Mol Sci. 2017 18: 2602-2612.

8. Scicchitano, $\mathrm{P}$ et. Al.:Nutraceuticals and dyslipidaemia: Beyond the common therapeutics. Journal of Functional Foods on Science 6, January 2014, Pages 11-32.

9. Adefegha, SA: Functional Foods and Nutraceuticals as Dietary Intervention in Chronic Diseases; Novel Perspectives for Health Promotion and Disease Prevention. J Diet Suppl. 2018 15: 977-1009.

10. Beisswenger P: Glycation and biomarkers of vascular complications of diabetes. Amino Acids 2012 42: 1171-1183.

11. Bucala R, Cerami A: Advanced Glycosylation: Chemistry, Biology, and Implications for Diabetes and Aging.

12. Singh VP, Bali A, Singh N, Jaggi, AS: Advanced Glycation End Products and Diabetic Complications. Korean J Physiol Pharmacol. 2014 18: 1-14.

13. Jafarnejad, A., Bathaie, S.Z., Nakhjavani, M., Hassan, M.Z., Banasadegh, S: The improvement effect of L-Lys as a chemical chaperone on STZ-induced diabetic rats, protein structure and function. Diabetes/Metabolism Research and Reviews. 2008 24: 64-73. 
14. Burd, J.F., F.A. Alvarez Malero, Nortzel, V: Hemoglobin A1c (HbA1c) shows improvement in glycemic control in as little as two weeks following the addition of Lysulin to the treatment of diabetes. Diabetes Management. 2018 8: 82-84.

15. Burd, J.F., Nortzel, V., Gonzales, A Alvarez Malero, F.A: Lysulin®: A double-blind placebo controlled pilot study of daily oral supplementation in people with type 2 diabetes. Diabetes Management. 2018 8: 154-162.

16. Jayawardena R, et.al: Effects of zinc supplementation on diabetes mellitus: A systematic review and meta-analysis. Diabetol Metab Syndr. 2012; 13 4-13.

17. Dakhale GN, Chaudhari HV, Shrivastava M: Supplementation of Vitamin C Reduces Blood Glucose and Improves Glycosylated Hemoglobin in Type 2 Diabetes Mellitus: A Randomized, Double-Blind Study. Advances in Pharmacological Sciences. 2011 5: 235-242.

18. Klonoff DC, et al: Clinical Evaluation of a Rapid A1C Test (A1cNow) for Home Use. Point Care 2006 5(3): 116-120.

19. Facchinetti, A: Continuous Glucose Monitoring Sensors: Past, Present and Future Algorithmic Challenges. Sensors. 2016 16, 1-12.

20. Burd JF, Noetzel N, Walsh MJ: Lysulin ${ }^{\mathrm{TM}}$ : Improvement in Glycemic Control Compared to Type 2 Drugs. Int J Diab Endocrinol 2019 1(1): 1-9. 